\title{
Handling the Low-frequency Breakdown of the PMCHWT Integral Equation with the quasi-Helmholtz Projectors
}

\author{
Yves Beghein* Rajendra Mitharwal ${ }^{\dagger}$
}

\author{
Kristof Cools $\ddagger \quad$ Francesco P. Andriulli ${ }^{\dagger}$
}

\begin{abstract}
This contribution presents a quasiHelmholtz projectors based regularization of the low frequency breakdown of the PMCHWT integral equation. The PMCHWT equation in the lowfrequency regime shows an ill-conditioned behavior inherited from the Electric Field Integral Operators it contains. The stabilization via quasi-Helmholtz projectors, differently from the use of standard Loop-Star/Tree decompositions, does not introduce an additional mesh-size-related ill-conditioning and it applies smoothly to both simply and non-simply connected geometries. The presentation of the main formulation will be complemented by numerical results demonstrating the effectiveness and accuracy of the proposed scheme.
\end{abstract}

\section{INTRODUCTION}

The Poggio-Miller-Chan-Harrington-Wu-Tsai (PMCHWT) integral equation is one of the main formulations for solving radiation and scattering problems for penetrable objects in the frequency domain [1].

The equation is of the first kind and, similarly to the electric field integral equation (EFIE) [2], it suffers from severe ill-conditioning problems when the simulation frequency is low. This is the well-known "low-frequency" breakdown phenomenon $[3,4]$. Historically, the breakdown can be solved by using quasi-Helmholtz decompositions such as Loop-Star and Loop-Tree basis functions [5, $6,7,8]$. These decompositions however, although solving the frequency-related ill-conditioning, introduce a parasitic and mesh-dependent form of illconditioning [9] since they can be interpreted as graph counterparts of differential operators [10].

Recently, a solution to this problem has been introduced for the EFIE based on newly introduced quasi-Helmholtz projectors [11]. These projectors realize a quasi-Helmholtz decomposition, but in an implicit and well-conditioned way, so that the un-

\footnotetext{
*Department of Information Technology (INTEC), Ghent University, Ghent, Belgium; e-mail: yves. beghein@ugent. be

†CERL Laboratory, Télécom Bretagne / Institut Mines-Télécom, Brest, France; e-mail: francesco.andriulli@mines-telecom.fr

${ }^{\ddagger}$ Electrical Systems and Optics Research Division, University of Nottingham, Nottingham, U.K.; e-mail: kristof.cools@nottingham.ac.uk
}

desired mesh-dependent ill-conditioning generated by standard approaches does not occur. The approach has been also successfully exploited in time domain [12].

This work generalizes this strategy to the treatment of the low-frequency breakdown of the PMCHWT integral equation. The generalization is challenging since, differently from the EFIE, the offdiagonal blocks of the PMCHWT requires a suitable analysis and treatment to ensure low-frequency stability.

Numerical results corroborates the theory and shows the practical applicability of the new approach.

\section{BACKGROUND AND NOTATION}

Consider a dielectric region $\Omega$ of intrinsic impedance $\eta_{k^{\prime}}$ with its external region $\Omega / \mathbb{R}^{3}$ of intrinsic impedance $\eta_{k}$. We denote with $\Gamma=\partial \Omega$ its boundary surface with outward directed normal $\hat{n}$. The Electric Field Integral Operator (EFIO) and Magnetic Field Integral Operator (MFIO) read

$$
\begin{aligned}
\mathcal{T}_{\alpha}(\boldsymbol{f}(\boldsymbol{r})) & =\hat{n} \times i \alpha \int_{\Gamma} \frac{e^{i \alpha\left|\boldsymbol{r}-\boldsymbol{r}^{\prime}\right|}}{4 \pi\left|\boldsymbol{r}-\boldsymbol{r}^{\prime}\right|} \boldsymbol{f}\left(\boldsymbol{r}^{\prime}\right) d \Gamma \\
& -\hat{n} \times \frac{1}{i \alpha} \nabla \int_{\Gamma} \frac{e^{i \alpha\left|\boldsymbol{r}-\boldsymbol{r}^{\prime}\right|}}{4 \pi\left|\boldsymbol{r}-\boldsymbol{r}^{\prime}\right|} \nabla^{\prime} \cdot \boldsymbol{f}\left(\boldsymbol{r}^{\prime}\right) d \Gamma
\end{aligned}
$$

$$
\mathcal{K}_{\alpha}(\boldsymbol{f}(\boldsymbol{r}))=-\hat{n} \times \int_{\Gamma} \nabla \frac{e^{i \alpha\left|\boldsymbol{r}-\boldsymbol{r}^{\prime}\right|}}{4 \pi\left|\boldsymbol{r}-\boldsymbol{r}^{\prime}\right|} \times \boldsymbol{f}\left(\boldsymbol{r}^{\prime}\right) d \Gamma,
$$

where the subscript $\alpha$ represents the wave number $k$ or $k^{\prime}$ for the region $\Omega / \mathbb{R}^{3}$ or $\Omega$ respectively. The PMCHWT equation models scattering from the penetrable region $\Omega$ when illuminated by an incident electromagnetic field $\left(\boldsymbol{E}_{i}(\boldsymbol{r}), \boldsymbol{H}_{i}(\boldsymbol{r})\right)$. It reads

$$
\begin{array}{r}
{\left[\begin{array}{cc}
\mathcal{T}_{k} / \eta_{k}+\mathcal{T}_{k^{\prime}} / \eta_{k^{\prime}} & -\left(\mathcal{K}_{k}+\mathcal{K}_{k^{\prime}}\right) \\
\left(\mathcal{K}_{k}+\mathcal{K}_{k^{\prime}}\right) & \left(\eta_{k} \mathcal{T}_{k}+\eta_{k^{\prime}} \mathcal{T}_{k^{\prime}}\right)
\end{array}\right]\left[\begin{array}{c}
\boldsymbol{M}(\boldsymbol{r}) \\
\boldsymbol{J}(\boldsymbol{r})
\end{array}\right]} \\
=\left[\begin{array}{l}
-\hat{n} \times \boldsymbol{H}_{i}(\boldsymbol{r}) \\
-\hat{n} \times \boldsymbol{E}_{i}(\boldsymbol{r})
\end{array}\right]
\end{array}
$$

where $\boldsymbol{J}(\boldsymbol{r})$ and $\boldsymbol{M}(\boldsymbol{r})$ represent the electric and magnetic currents, respectively. 
On each edge of the triangular surface mesh approximating the surface $\Gamma$, an RWG basis function $\boldsymbol{f}_{n}(\boldsymbol{r})$ is defined [2]. This set approximates the currents as $\boldsymbol{J}(\boldsymbol{r})=\sum_{n=1}^{N} \alpha_{n} \boldsymbol{f}_{n}(\boldsymbol{r})$ and $\boldsymbol{M}(\boldsymbol{r})=$ $\sum_{n=1}^{N} \beta_{n} \boldsymbol{f}(\boldsymbol{r})$. The overall discretized PMCHWT equation is given by

$$
\left[\begin{array}{cc}
\mathbf{T}_{k} / \eta_{k}+\mathbf{T}_{k^{\prime}} / \eta_{k^{\prime}} & -\left(\mathbf{K}_{k}+\mathbf{K}_{k^{\prime}}\right) \\
\left(\mathbf{K}_{k}+\mathbf{K}_{k^{\prime}}\right) & \left(\eta_{k} \mathbf{T}_{k}+\eta_{k^{\prime}} \mathbf{T}_{k^{\prime}}\right)
\end{array}\right]\left[\begin{array}{c}
\beta \\
\alpha
\end{array}\right]=\left[\begin{array}{c}
\mathbf{h} \\
\mathbf{e}
\end{array}\right]
$$

where, element-wise, $\quad\left(\mathbf{T}_{k}\right)_{m n}=$ $\left\langle\hat{n} \times \boldsymbol{f}_{m}(\boldsymbol{r}), \mathcal{T}_{k}\left(\boldsymbol{f}_{n}(\boldsymbol{r})\right)\right\rangle_{\Gamma}$ using the notation $\langle\boldsymbol{a} \cdot \boldsymbol{b}\rangle_{\chi}=\int_{\chi} \boldsymbol{a} \cdot \boldsymbol{b} d \chi\left(\mathbf{T}_{k^{\prime}}, \mathbf{K}_{k}\right.$ and $\mathbf{K}_{k}$ are similarly defined). The right hand side (RHS) vectors are given by $\mathbf{h}_{m}=\left\langle\hat{n} \times \boldsymbol{f}_{m}(\boldsymbol{r}), \boldsymbol{H}_{i}(\boldsymbol{r})\right\rangle_{\Gamma}$ and $\mathbf{e}_{m}=\left\langle\hat{n} \times \boldsymbol{f}_{m}(\boldsymbol{r}), \boldsymbol{E}_{i}(\boldsymbol{r})\right\rangle_{\Gamma}$. The EFIO blocks on the diagonal of the system matrix in equation (4) suffer from the low-frequency breakdown, a problem which is consequently transferred to the entire PMCHWT equation.

\section{PROPOSED REGULARIZATION}

To properly regularize the effect of low frequency breakdown in equation (4), it is useful to separate the different contributions in the EFIO

$$
\begin{aligned}
\mathbf{T}_{\boldsymbol{A}}^{a} & =\frac{1}{k}\left(\mathbf{T}_{k}^{\boldsymbol{A}} / \eta_{k}+\mathbf{T}_{k^{\prime}}^{\boldsymbol{A}} / \eta_{k^{\prime}}\right) \\
\mathbf{T}_{\phi}^{a} & =k\left(\mathbf{T}_{k}^{\phi} / \eta_{k}+\mathbf{T}_{k^{\prime}}^{\phi} / \eta_{k^{\prime}}\right) \\
\mathbf{T}_{\boldsymbol{A}}^{b} & =\frac{1}{k}\left(\mathbf{T}_{k}^{\boldsymbol{A}} \eta_{k}+\mathbf{T}_{k^{\prime}}^{\boldsymbol{A}} \eta_{k^{\prime}}\right) \\
\mathbf{T}_{\phi}^{b} & =k\left(\mathbf{T}_{k}^{\phi} \eta_{k}+\mathbf{T}_{k^{\prime}}^{\phi} \eta_{k^{\prime}}\right)
\end{aligned}
$$

where the subscripts $\phi$ and $\boldsymbol{A}$ represent the scalar and vector potential term respectively (the first and second term on the RHS of equation (1)). The MFIO matrix should also be decomposed as

$$
\begin{aligned}
& \mathbf{K}_{z}=2 \mathbf{K}_{0} \\
& \mathbf{K}_{e}=\mathbf{K}_{k}+\mathbf{K}_{k^{\prime}}-\mathbf{K}_{z}
\end{aligned}
$$

where $\mathbf{K}_{0}$ is the static MFIO matrix corresponding to $k=0$. Using equation (5) and (6), the system matrix in the equation (4) can be written as

$$
\left[\begin{array}{cc}
k \mathbf{T}_{\boldsymbol{A}}^{a}+\frac{1}{k} \mathbf{T}_{\phi}^{a} & -\left(\mathbf{K}_{e}+\mathbf{K}_{z}\right) \\
\mathbf{K}_{e}+\mathbf{K}_{z} & k \mathbf{T}_{\boldsymbol{A}}^{b}+\frac{1}{k} \mathbf{T}_{\phi}^{b}
\end{array}\right] .
$$

The objective behind these matrix decompositions is to have a low-frequency-stable behavior of the conditioning of the overall system matrix after applying the quasi-Helmholtz projectors. These projectors can be defined using the loop/star-to-RWGs transformation matrices $\boldsymbol{\Sigma}$ and $\boldsymbol{\Lambda}$ (we omit the definition of these matrices for space limitations, the reader is invited to refer to [11] for an exhaustive definition) as $\mathbf{P}^{\Sigma}=\boldsymbol{\Sigma}\left(\boldsymbol{\Sigma} \boldsymbol{\Sigma}^{T}\right)^{+} \boldsymbol{\Sigma}^{T}$ and $\mathbf{P}^{\Lambda H}=\mathbf{I}-\boldsymbol{\Sigma}\left(\boldsymbol{\Sigma} \boldsymbol{\Sigma}^{T}\right)^{+} \boldsymbol{\Sigma}^{T}$ on the surface mesh and its dual counterparts $\mathbb{P}^{\Lambda}=\boldsymbol{\Lambda}\left(\boldsymbol{\Lambda} \boldsymbol{\Lambda}^{T}\right)^{+} \boldsymbol{\Lambda}^{T}$ and $\mathbb{P}^{\Sigma H}=\mathbf{I}-\boldsymbol{\Lambda}\left(\boldsymbol{\Lambda} \boldsymbol{\Lambda}^{T}\right)^{+} \boldsymbol{\Lambda}^{T}$ on the dual mesh [11]. The decomposition operator matrix is defined as $\mathbf{M}=\mathbf{P}^{\Lambda H} \frac{1}{\sqrt{k}}+i \mathbf{P}^{\Sigma} \sqrt{k}$. Similarly, the associated dual decomposition operator matrix is defined as $\mathbb{M}=\mathbb{P}^{\Lambda} \frac{1}{\sqrt{k}}+i \mathbb{P}^{\Sigma H} \sqrt{k}$. The associated Gram matrix linking the basis functions on the primal and the dual mesh is defined as $\left[\mathbf{G}_{m i x}\right]_{m n}=$ $\left\langle\boldsymbol{f}_{m}(\boldsymbol{r}), \hat{\boldsymbol{n}}_{\boldsymbol{r}} \times \boldsymbol{f}_{n}^{B C}(\boldsymbol{r})\right\rangle_{\Gamma}$ where $\left\{\boldsymbol{f}_{n}^{B C}(\boldsymbol{r})\right\}_{n=1}^{N}$ is the set of Buffa-Christiansen (BC) basis functions [13] defined on the dual mesh.

The proposed regularization based on the quasiHelmholtz projectors for the EFIO blocks in the equation (7) reads

$$
\begin{aligned}
& \mathbb{M}^{T} \mathbf{G}_{m i x}^{-1} \mathbf{T}^{x} \mathbf{M}=\left(\mathbb{P}^{\Lambda} \mathbf{G}_{m i x}^{-1} \mathbf{T}_{A}^{x} \mathbf{P}^{\Lambda H}-\mathbb{P}^{\Sigma H} \mathbf{G}_{m i x}^{-1} \mathbf{T}_{\phi}^{x} \mathbf{P}^{\Sigma}\right) \\
& +i k\left(\mathbb{P}^{\Lambda} \mathbf{G}_{m i x}^{-1} \mathbf{T}_{A}^{x} \mathbf{P}^{\Sigma}+\mathbb{P}^{\Sigma H} \mathbf{G}_{m i x}^{-1} \mathbf{T}_{A}^{x} \mathbf{P}^{\Lambda H}\right) \\
& -k^{2} \mathbb{P}^{\Sigma H} \mathbf{G}_{m i x}^{-1} \mathbf{T}_{\boldsymbol{A}}^{x} \mathbf{P}^{\Sigma}
\end{aligned}
$$

where the superscript $x$ can be either $a$ or $b$ depending on the diagonal block matrix. In order to simplify the above expression, we have used $\mathbf{T}_{\phi}^{x} \mathbf{P}^{\Lambda H}=\mathbf{0}$ and $\mathbb{P}^{\Lambda} \mathbf{G}_{m i x}^{-1} \mathbf{T}_{\phi}^{x}=\mathbf{0}$. Along the same lines, the proposed regularization for the MFIO blocks is

$$
\begin{aligned}
& \mathbb{M}^{T} \mathbf{G}_{m i x}^{-1} \mathbf{K M}=\frac{1}{k} \mathbb{P}^{\Lambda} \mathbf{G}_{m i x}^{-1} \mathbf{K}_{e} \mathbf{P}^{\Lambda H}+i\left(\mathbb{P}^{\Lambda} \mathbf{G}_{m i x}^{-1} \mathbf{K}_{z} \mathbf{P}^{\Sigma}\right. \\
& +\mathbb{P}^{\Lambda} \mathbf{G}_{m i x}^{-1} \mathbf{K}_{e} \mathbf{P}^{\Sigma}+\mathbb{P}^{\Sigma H} \mathbf{G}_{m i x}^{-1} \mathbf{K}_{z} \mathbf{P}^{\Lambda H} \\
& \left.+\mathbb{P}^{\Sigma H} \mathbf{G}_{m i x}^{-1} \mathbf{K}_{e} \mathbf{P}^{\Lambda H}\right)-k\left(\mathbb{P}^{\Sigma H} \mathbf{G}_{m i x}^{-1} \mathbf{K}_{e} \mathbf{P}^{\Sigma}\right. \\
& \left.+\mathbb{P}^{\Sigma H} \mathbf{G}_{m i x}^{-1} \mathbf{K}_{z} \mathbf{P}^{\Sigma}\right)
\end{aligned}
$$

where we have used the property of static MFIE operator matrix as $\mathbb{P}^{\Lambda} \mathbf{G}_{m i x}^{-1} \mathbf{K}_{z} \mathbf{P}^{\Lambda H}=0$.

The proposed quasi-Helmholtz projected PMCHWT system, where the new unknown vectors are $\tilde{\alpha}=\mathbf{M}^{-1} \alpha$ and $\tilde{\beta}=\mathbf{M}^{-1} \beta$, reads

$$
\left[\begin{array}{ll}
\mathbf{Z}_{11} & \mathbf{Z}_{12} \\
\mathbf{Z}_{21} & \mathbf{Z}_{22}
\end{array}\right]\left[\begin{array}{l}
\tilde{\alpha} \\
\tilde{\beta}
\end{array}\right]=\left[\begin{array}{l}
\mathbf{h}_{p} \\
\mathbf{e}_{p}
\end{array}\right]
$$

where $\mathbf{Z}_{11}=\mathbb{M}^{T} \mathbf{G}_{m i x}^{-1}\left(k \mathbf{T}_{\boldsymbol{A}}^{a}+\frac{1}{k} \mathbf{T}_{\phi}^{a}\right) \mathbf{M}, \mathbf{Z}_{12}=$ $\left.-\mathbb{M}^{T} \mathbf{G}_{m i x}^{-1}\left(\mathbf{K}_{e}+\mathbf{K}_{z}\right)\right) \mathbf{M}, \mathbf{Z}_{21}=\mathbb{M}^{T} \mathbf{G}_{m i x}^{-1}\left(\mathbf{K}_{e}+\right.$ $\left.\mathbf{K}_{z}\right) \mathbf{M}$ and $\mathbf{Z}_{22}=\mathbb{M}^{T} \mathbf{G}_{m i x}^{-1}\left(k \mathbf{T}_{\boldsymbol{A}}^{b}+\frac{1}{k} \mathbf{T}_{\phi}^{b}\right) \mathbf{M}$.

\section{NUMERICAL RESULTS}

The proposed regularization is applied to a scenario involving scattering due to a homogeneous dielec- 
tric sphere of the radius $1 \mathrm{~m}$ with relative permittivity as $\epsilon_{r}=3$. The electromagnetic source is a plane wave travelling in the $z$ direction and is polarized along the $x$ axis with an electric field intensity of $1 \mathrm{~V} / \mathrm{m}$. The condition number of the regularized system matrix stays constant when compared to the original PMCHWT matrix as shown in Figure 1 when the frequency decreases from $1 \mathrm{MHz}$ to $10^{-40} \mathrm{~Hz}$. The far field computed using the proposed regularization shows a good agreement with the Mie series in Figure 2 and 3 for the frequency $1 \mathrm{MHz}$ and $10^{-40} \mathrm{~Hz}$ respectively.

In a second example, a torus model with the centerline radius of $3 \mathrm{~m}$ and the cross-section radius of $0.5 \mathrm{~m}$ with relative permittivity as $\epsilon_{r}=3$ is chosen. The parameters for the electromagnetic source are kept equal to that of the sphere. The condition number of the regularized system matrix stays constant as seen in Figure 4 when the frequency is decreased till $10^{-40} \mathrm{~Hz}$. The far field due to both the original and regularized formulation shows a perfect matching in Figure 5 for a frequency of $10 \mathrm{MHz}$.

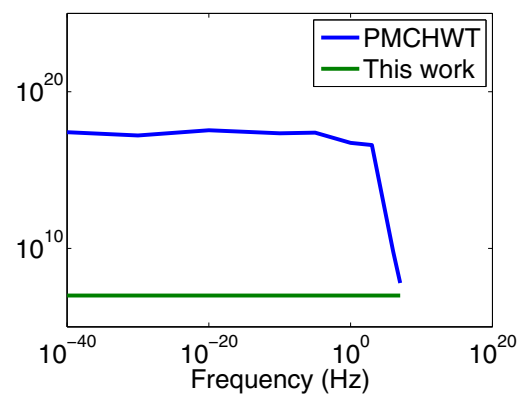

Figure 1: Sphere: Condition number w.r.t to frequency.

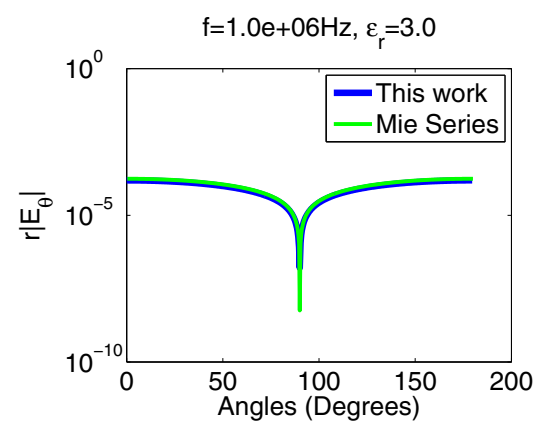

Figure 2: Sphere: Far field calculated at frequency of $1 \mathrm{MHz}$.

\section{CONCLUSION}

We have presented a quasi-Helmholtz projectors based regularization for the system matrix arising

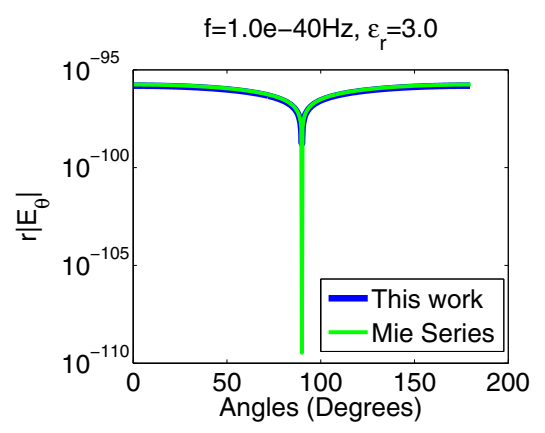

Figure 3: Sphere: Far field calculated at frequency of $10^{-40} \mathrm{~Hz}$.

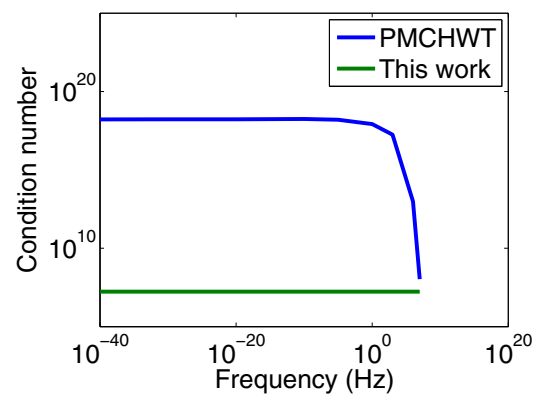

Figure 4: Torus: Condition number w.r.t to frequency.

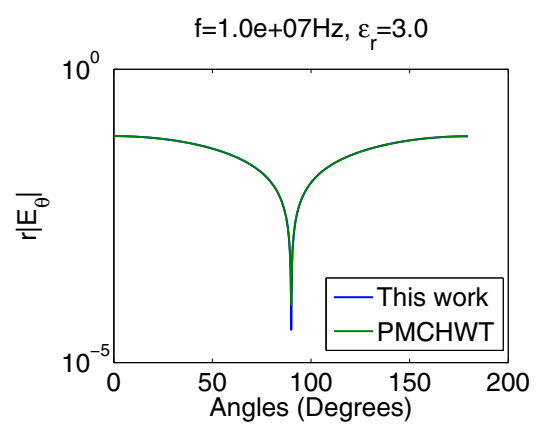

Figure 5: Torus: Far field calculated at frequency of $10 \mathrm{MHz}$. 
from the discretization of the PMCHWT integral equations. The proposed regularization ensures a frequency independent behavior for the condition number of the system matrix. Numerical results demonstrate the accuracy and applicability of the new scheme to both simply and non-simply connected geometries.

\section{Acknowledgments}

This work was supported in part by the Agence Nationale de la Recherche under Project FASTEEGANR-12-JS09-0010, in part by the European Union Marie Curie Project NEUROIMAGEEG, in part by the Brittany Region, France, in part by the HPC resources through the GENCI-TGCC Project under Grant 2015-gen6944, and in part by a doctoral grant from the Agency for Innovation by Science and Technology in Flanders (IWT).

\section{References}

[1] W. C. Chew, E. Michielssen, J. Song, and J. Jin, Fast and efficient algorithms in computational electromagnetics. Artech House, Inc., 2001.

[2] S. M. Rao, D. Wilton, and A. W. Glisson, "Electromagnetic scattering by surfaces of arbitrary shape," IEEE TAP, vol. 30, no. 3, 1982.

[3] J.-S. Zhao and W. C. Chew, "Integral equation solution of maxwell's equations from zero frequency to microwave frequencies," IEEE TAP, vol. 48, no. 10, pp. 1635-1645, 2000.

[4] J.-F. Lee, J.-F. Lee, and R. J. Burkholder, "Loop star basis functions and a robust preconditioner for efie scattering problems," IEEE TAP, vol. 51, no. 8, pp. 1855-1863, 2003.

[5] D. Wilton and A. Glisson, "On improving the electric field integral equation at low frequencies," Proc. URSI Radio Sci. Meet. Dig, vol. $24,1981$.

[6] M. Burton and S. Kashyap, "A study of a recent, moment-method algorithm that is accurate to very low frequencies," ACES Journal, vol. 10, pp. 58-68, 1995.

[7] W.-L. Wu, A. W. Glisson, and D. Kajfez, "A study of two numerical solution procedures for the electric field integral equation at low frequency," ACES Journal, vol. 10, no. 3, 1995.
[8] G. Vecchi, "Loop-star decomposition of basis functions in the discretization of the efie," IEEE TAP, vol. 47, no. 2, pp. 339-346, 1999.

[9] F. P. Andriulli, "Loop-star and loop-tree decompositions: Analysis and efficient algorithms," IEEE TAP, vol. 60, no. 5, 2012.

[10] F. P. Andriulli, A. Tabacco, and G. Vecchi, "Solving the efie at low frequencies with a conditioning that grows only logarithmically with the number of unknowns," IEEE TAP, vol. 58, no. 5, 2010 .

[11] F. Andriulli, K. Cools, I. Bogaert, and E. Michielssen, "On a well-conditioned electric field integral operator for multiply connected geometries," IEEE TAP, vol. 61, no. 4, 2013.

[12] Y. Beghein, K. Cools, and F. Andriulli, "A dc stable and large time step well-balanced td-efie based on quasi-helmholtz projectors," IEEE TAP, 2015.

[13] F. P. Andriulli, K. Cools, H. Bagci, F. Olyslager, A. Buffa, S. Christiansen, and E. Michielssen, "A multiplicative calderon preconditioner for the electric field integral equation," IEEE TAP, vol. 56, no. 8, 2008. 